\title{
The location-routing problem under emergency logistics in post-earthquake
}

\author{
Changshi Liu'1,2,3 \\ ${ }^{1}$ School of Management and Economics, University of Electronic Science and Technology of China, \\ Chengdu, 611731, China; \\ ${ }^{2}$ Hunan University of Commerce, Changsha 410205, China \\ ${ }^{3}$ Key Laboratory of Hunan Province for Mobile Business Intelligence, Hunan University of \\ Commerce, Changsha 410205, China
}

liuchangshi@hnuc.edu.cn

Keywords: earthquake; emergency logistics; relief delivery; location-routing problem

Abstract. The location of distribution centers and routing of relief delivery in the available transportation network are two of the most challenging issues in emergency logistics after disasters. This paper presents a stochastic mixed-integer program model that integrates fuzzy demands, time windows, and damage of emergency logistics network into an integrated location-routing problem (LRP). A hybrid heuristics is designed to solve the proposed LRP. Numerical experiments show that the proposed model and solution approach are effective and reasonable.

\section{Introduction}

Frequent earthquake events highlight the need for a reliable and responsive emergency logistics that can effectively mitigate the adverse impacts. It is a challenge to properly delivery relief to affected areas in uncertain situations[1]. The location of distribution centers and routing of relief delivery in the available transportation network are two of the most challenging issues in emergency logistics after disasters. And the two rely on each other and influence each other. So, the LRP in emergency logistics system after earthquake must be studied[2].

Now, LRP attracts increasing attention. Hu and Sheng used a state diagram to construct a microscopic behavior model for the resource nodes by regarding the resource nodes as agents[3]. Sheu designed a dynamic relief-demand management plan for emergency logistics operations under large-scale disasters[4]. Rath and Gutjahr considered a math-heuristic for the warehouse LRP in disaster relief, and they formulated a three-objective optimization model with a medium-term economic, a short term economic, and a humanitarian objective function[5]. Lin et al. proposed the location of temporary depots around the disaster-affected area, along with the required vehicles and resources, to improve logistical efficiency[6]. Ahmadi et al. proposed a LRP model considering network failure, multiple uses of vehicles, and standard relief time[7].

This paper presents a mixed-integer program LRP model by considering fuzzy demands, time windows, and damage of emergency logistics network. A heuristics is designed to solve the LRP.

\section{Problem statement}

It is assumed that the distribution depots must be selected from the potential depots at the initial deployment phase of emergency logistics operation in post-earthquake before delivering supplies to victims. The potential depots are usually located at existing buildings or simply placed on vacant ground which near the earthquake-affected areas. The distribution depots receive reliefs from the external emergency supply agents within the deployment stage and deliver reliefs to allocated demand points immediately after the earthquake. 


\section{LRP model}

\section{Notation, parameters and variables}

$A$--the set of potential distribution depots; $w_{g}$--the capacity of potential distribution depot $g ; B$--the set of demand points; $d_{h}$--the fuzzy demand of point $h, d_{h}=\left(d_{h 1}, d_{h 2}, d_{h 3}\right)$; $\left[\varpi_{h s}, \varpi_{h e}\right]$--the time window of point $h ; N=A \bigcup B$--the set of all nodes, $i, j \in N ; c_{i j}$--the distance between node $i$ and node $j ; V$--the set of vehicles; $Q_{k}$--the capacity of vehicle $k ; r_{k}$--the speed of vehicle $k ; t_{i j k}$--travel time of vehicle $k$ from node $i$ to node $j, t_{i j k}=c_{i j} / r_{k} ; T_{i k}$-- the time of vehicle $k$ arriving at node $i$, when $i \in A, T_{i k}=0$.

$x_{g}$--binary variable that equals 1 if depot $g$ is selected, and 0 otherwise; $y_{g k}$--binary variable that equals 1 if vehicle $k$ is allocated to depot $g$, and 0 otherwise; $z_{i j k}-$ binary variable that equals 1 if vehicle $k$ travel from node $i$ to node $j$. $a_{i j}$--binary variable that equals 1 if node $i$ connects node $j$, and 0 otherwise.

\section{LRP formulation}

$\min f=\sum_{k \in V} \sum_{i \in N} T_{i k}$

s.t. $\sum_{i \in N} \sum_{h \in B} z_{i h k} d_{h}^{*} \leq Q_{\mathrm{k}}, \forall k \in V$

$\sum_{i \in N} \sum_{h \in B} \sum_{k \in V} z_{i h k} d_{h}^{*} y_{g k} \leq w_{g} x_{\mathrm{g}}, \forall g \in A$

$\sum_{h \in B} \sum_{k \in V} z_{g h k}-x_{g} \geq 0, \forall g \in A$

$\sum_{h \in B} z_{g h k}-y_{g k} \leq 0, \forall g \in A, \forall k \in V$

$\sum_{k \in V} z_{g l k}=0, \quad \forall g, l \in A$

$\sum_{i \in N} z_{i j k}-\sum_{i \in N} z_{j i k}=0, \quad \forall k \in V, j \in N$

$\sum_{i \in N} \sum_{k \in V} z_{i h k}=1, \quad \forall h \in B$

$T_{j k}=T_{i k}+t_{i j k} z_{i j k}, \forall i \in N, \forall j \in N, \forall k \in V$

$\varpi_{h s} \leq T_{j k} \leq \varpi_{h e}, \forall h \in B, \forall j \in B, \forall k \in V$

$x_{g} \in\{0,1\}, y_{g k} \in\{0,1\}, z_{i j k} \in\{0,1\}$

which $d_{h}^{*}$ is the actual demand obtained by the fuzzy number expected value method, $d_{h}^{*}=\left(d_{h 1}+2 d_{h 2}+d_{h 3}\right) / 4$.

The objective function of the LRP model, as shown in (1), states that the goal is to minimize the total delivery time. Constraint (2) ensures that reliefs of each vehicle do not exceed the capacity limit. Constraint (3) represents the capacity limit of distribution depot. Constraint (4) ensures that the selected depots have vehicle for delivery relief. Constraint (5) ensures that unselected depots have no vehicle. Constraint (6) ensures that any two selected depots can not be on the same path. Constraint (7) ensures the path continuity of vehicle. Constraint (8) ensures that each demand node is visited by only one vehicle. Constraint (9) represents the time calculation method of vehicle arriving at demand point. Constraint (10) represents the time window. Constraint (11) represents constraint of variable value. 


\section{Hybrid heuristics}

This paper designs a global hybrid heuristics for the LRP. The details are described as follows:

Step 1: Variable initialization. Input values for all variables, set the value of max iteration, let the value of currentiteration, and let the total delivery time $f=\infty$.

Step 2: Allocation of demand points. The greedy algorithm is used to randomly allocate the demand points to distribution depots with the capacity constraints.

Step 3: Distribution mode selection of demand points. If $a_{i j}=1$, the relief of the demand point is distributed by vehicle; else, by helicopter.

Step 4: Routing plan. For each selected distribution depots, the ant colony algorithm ${ }^{[8]}$ is adopted to optimize the vehicle routing and helicopter routing. The total delivery time of current iteration $f_{\text {iter }}$ is calculated. If $f_{\text {iter }}<f$, let $f=f_{\text {iter }}$.

Step 5: Algorithm termination. If currentiteration $<\max$ iteration, currentiteration $=$ currentiteration +1 , return to step 2; else, algorithm termination.

\section{Computational results}

It is assumed that there are 6 potential depots in the earthquake zone. The coordinates and capacities of the potential depots are listed in Table 1. The detail of vehicles and helicopters are shown in Table 2. The coordinate, demands and time limits of demand points are described in Table 3. For simplicity, the connectivity between nodes is omitted. The program for our global hybrid heuristics $(\mathrm{GHH})$ was developed in Matlab 7.0.

Table 1. The coordinates and capacities of the potential depots

\begin{tabular}{|c|c|c|c|c|c|c|}
\hline number & 1 & 2 & 3 & 4 & 5 & 6 \\
\hline coordinate & $(35,26)$ & $(78,92)$ & $(46,34)$ & $(64,81)$ & $(91,68)$ & $(77,59)$ \\
\hline capacity & 3000 & 4500 & 4500 & 4400 & 4000 & 4500 \\
\hline
\end{tabular}

Table 2. The detail of vehicles and helicopters

\begin{tabular}{|c|c|c|}
\hline & capacity & speed \\
\hline vehicle & 600 & 50 \\
\hline helicopter & 1200 & 500 \\
\hline
\end{tabular}

Table 3. The demands and time limits of demand points

\begin{tabular}{|c|c|c|c|c|c|}
\hline number & 1 & 2 & 3 & 4 & 5 \\
\hline coordinate & $(11,22)$ & $(14,37)$ & $(22,44)$ & $(41,51)$ & $(72,81)$ \\
\hline demand & $(350,400,450)$ & $(250,300,350)$ & $(310,380,430)$ & $(350,400,450)$ & $(350,400,440)$ \\
\hline time limit & 300 & 250 & 350 & 400 & 400 \\
\hline number & 6 & 7 & 8 & 9 & 10 \\
\hline coordinate & $(91,36)$ & $(51,62)$ & $(44,77)$ & $(82,14)$ & $(98,66)$ \\
\hline demand & $(350,400,440)$ & $(350,400,450)$ & $(250,300,350)$ & $(310,380,430)$ & $(250,300,350)$ \\
\hline time limit & 350 & 300 & 250 & 350 & 400 \\
\hline number & 11 & 12 & 13 & 14 & 15 \\
\hline coordinate & $(55,81)$ & $(22,33)$ & $(40,20)$ & $(50,30)$ & $(60,40)$ \\
\hline demand & $(360,400,440)$ & $(350,400,440)$ & $(550,600,650)$ & $(650,700,750)$ & $(800,900,980)$ \\
\hline time limit & 400 & 350 & 300 & 250 & 350 \\
\hline number & 16 & 17 & 18 & 19 & 20 \\
\hline coordinate & $(70,30)$ & $(50,60)$ & $(70,10)$ & $(87,26)$ & $(15,45)$ \\
\hline demand & $(350,400,440)$ & $(350,400,440)$ & $(350,400,440)$ & $(550,600,650)$ & $(650,700,750)$ \\
\hline time limit & 400 & 400 & 350 & 300 & 250 \\
\hline number & 21 & 22 & 23 & 24 & \\
\hline coordinate & $(39,89)$ & $(9,66)$ & $(44,77)$ & $(22,11)$ & \\
\hline demand & $(200,300,380)$ & $(150,200,250)$ & $(250,300,350)$ & $(150,200,250)$ & \\
\hline time limit & 350 & 400 & 400 & 350 & \\
\hline
\end{tabular}


The plan of the LRP is given in Figure 1. We can see that there are many directdistribution routes of full loaded vehicles, routes of travelling vehicles and routes of travelling helicopters. This shows that it is very important for the decision-maker to use a variety of distribution methods for delivering reliefs in post-earthquake. The directdistribution routes show that there are many damand nodes which need large quantity reliefs because that earthquake always causes serious consequences.
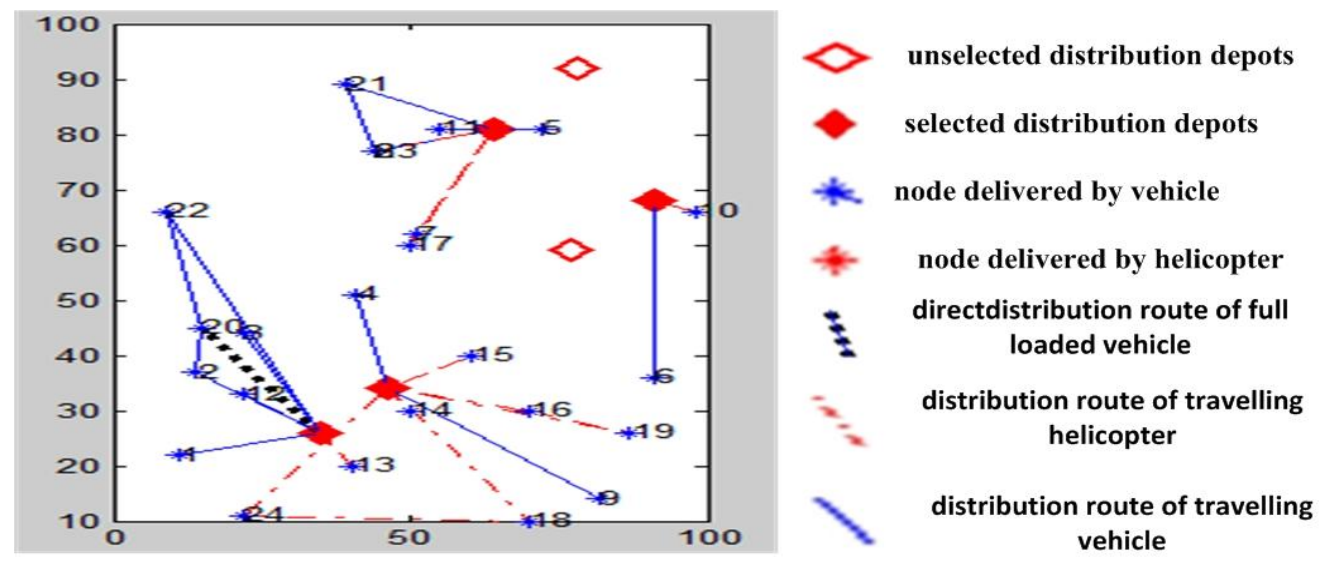

Figure 1. LRP plan

\section{Conclusions}

Earthquakes often bring huge disastrous consequences to human beings. In order to effectively carry out the earthquake relief work, the emergency supplies must be transported to the demand nodes in the shortest possible time. In this paper, a stochastic mixed-integer program LRP model by considering fuzzy demands, time windows, damage of emergency logistics network and stochastic travel time. A hybrid heuristics is designed to solve the proposed LRP. Numerical experiments show that the proposed model and solution approach are effective and reasonable.

\section{References}

[1] J. Ruan, P. Shi, C. Lim, etal. Relief supplies allocation and optimization by interval and fuzzy number approaches[J]. Information Sciences, Vol. 303(2015), p.15-32.

[2] H.Wang, L. Du, S. Ma. Multi-objective open location-routing model with split delivery for optimized relief distribution in post-earthquake[J]. Transportation Research Part E, Vol. 69(2014), p.160-179.

[3] Z. Hu, Z. Sheng. Disaster spread simulation and rescue time optimization in a resource network[J]. Information Sciences, Vol. 298( 2015), p.118-135.

[4] JB. Sheu, Dynamic relief-demand management for emergency logistics operations under large-scale disasters[J]. Transportation Research Part E, Vol. 46(2010) , p.1-17.

[5] S. Rath, W.J. Gutjahr. A math-heuristic for the warehouse location-routing problem in disaster relief[J]. Computers \& Operations Research, Vol. 42(2014), p.25-39.

[6] Y. Lin, R. Batta, P.A. Rogerson, A.B.M. Flanigan. Location of temporary depots to facilitate relief operations after an earthquake[J]. Socio-Economic Planning Sciences, Vol. 46(2012), p.112-123.

[7] M. Ahmadi, A. Seifi, B. Tootooni. A humanitarian logistics model for disaster relief operation considering network failure and standard relief time: A case study on San Francisco district[J]. Transportation Research Part E, Vol. 75(2015), p.145-163. 
[8] D. Martens, M. D. Backer, R. Haesen, etal. Classification with Ant Colony Optimization[J]. IEEE Transactions on Evolutionary Computation, Vol. 11(2007), p. 651-665. 